\title{
PIC SIMULATIONS OF THE EFFECT OF VELOCITY SPACE INSTABILITIES ON ELECTRON VISCOSITY AND THERMAL CONDUCTION
}

\author{
Mario A. Riquelme ${ }^{1}$, Eliot Quataert ${ }^{2}$, and Daniel Verscharen ${ }^{3}$ \\ ${ }^{1}$ Departamento de Física, Facultad de Ciencias Físicas y Matemáticas, Universidad de Chile, Chile; mario.riquelme@dfi.uchile.cl \\ ${ }^{2}$ Astronomy Department and Theoretical Astrophysics Center, University of California, Berkeley, CA 94720, USA; eliot@berkeley.edu \\ ${ }^{3}$ Space Science Center and Department of Physics, University of New Hampshire, Durham, NH 03824, USA; Daniel.Verscharen@unh.edu \\ Received 2016 February 7; revised 2016 May 10; accepted 2016 May 13; published 2016 June 21
}

\begin{abstract}
In low-collisionality plasmas, velocity-space instabilities are a key mechanism providing an effective collisionality for the plasma. We use particle-in-cell (PIC) simulations to study the interplay between electron- and ion-scale velocity-space instabilities and their effect on electron pressure anisotropy, viscous heating, and thermal conduction. The adiabatic invariance of the magnetic moment in low-collisionality plasmas leads to pressure anisotropy, $\Delta p_{j} \equiv p_{\perp, j}-p_{\|, j}>0$, if the magnetic field $\boldsymbol{B}$ is amplified ( $p_{\perp, j}$ and $p_{\|, j}$ denote the pressure of species $j$ (electron, ion) perpendicular and parallel to $\boldsymbol{B}$ ). If the resulting anisotropy is large enough, it can in turn trigger small-scale plasma instabilities. Our PIC simulations explore the nonlinear regime of the mirror, IC, and electron whistler instabilities, through continuous amplification of the magnetic field $|\boldsymbol{B}|$ by an imposed shear in the plasma. In the regime $1 \lesssim \beta_{j} \lesssim 20\left(\beta_{j} \equiv 8 \pi p_{j} /|\boldsymbol{B}|^{2}\right)$, the saturated electron pressure anisotropy, $\Delta p_{\mathrm{e}} / p_{\|, \mathrm{e}}$, is determined mainly by the (electron-lengthscale) whistler marginal stability condition, with a modest factor of $\sim 1.5-2$ decrease due to the trapping of electrons into ion-lengthscale mirrors. We explicitly calculate the mean free path of the electrons and ions along the mean magnetic field and provide a simple physical prescription for the mean free path and thermal conductivity in low-collisionality $\beta_{j} \gtrsim 1$ plasmas. Our results imply that velocity-space instabilities likely decrease the thermal conductivity of plasma in the outer parts of massive, hot, galaxy clusters. We also discuss the implications of our results for electron heating and thermal conduction in low-collisionality accretion flows onto black holes, including Sgr A* in the Galactic Center.
\end{abstract}

Key words: accretion, accretion disks - instabilities - plasmas - solar wind

\section{INTRODUCTION}

In a low collisionality plasma, differences in pressure along $\left(p_{\|, j}\right)$ and perpendicular $\left(p_{\perp, j}\right)$ to the local magnetic field $\boldsymbol{B}$ are produced by compression, shearing, and/or heating of the plasma. This is a consequence of the adiabatic invariance of the "bounce invariant" and the magnetic moment, $\mu_{j} \equiv v_{\perp, j}^{2} / B$, where $v_{\perp, j}$ is the velocity perpendicular to the local magnetic field, $B=|\boldsymbol{B}|$ and $j$ denotes the particle species (Kulsrud 1983). Thus, absent Coulomb collisions, magnetic field amplification and/or plasma compression generically drive $p_{\perp, j}>p_{\|, j}$, while a decrease in the magnetic field strength and/or plasma expansion generically drives $p_{\perp, j}<p_{\|, j}$. These pressure anisotropies can be dynamically important because they modify the effective magnetic tension in the plasma, produce an effective viscosity, and can drive velocity-space instabilities. Systems where $p_{\perp_{j}} \neq p_{\|_{j}}$ is believed to be important are low-luminosity accretion flows around compact objects (Sharma et al. 2007), the intracluster medium (Schekochihin et al. 2005; Lyutikov 2007), and the heliosphere (Maruca et al. 2011; Remya et al. 2013).

When $p_{\perp, \mathrm{e}}>p_{\|, \mathrm{e}}$, the electron whistler instability is excited and can limit the amount of electron pressure anisotropy that develops (Gary \& Wang 1996). Although the linear behavior of the whistler instability is well understood, the long term, nonlinear evolution of the instability is less clear. Indeed, in many astrophysically relevant cases, the generation of pressure anisotropy occurs over timescales longer than the initial exponential growth phase that characterizes velocity-space instabilities. Moreover, the ions are expected to develop their own pressure anisotropy in synch with the electrons, giving rise to analogous instabilities. For example, when $B$ grows, the mirror and ion- cyclotron (IC) instabilities regulate the ion pressure anisotropy (Hasegawa 1969; Gary 1992; Southwood \& Kivelson 1993).

Kunz et al. (2014) (using hybrid PIC) and Riquelme et al. (2015) (using full PIC with ion to electron mass ratios $m_{\mathrm{i}}$ / $\left.m_{\mathrm{e}}=1-10\right)$ studied the saturation of ion velocity-space instabilities in a model problem where background velocity shear amplifies/reduces the strength of a background magnetic field, thus continually driving pressure anisotropy. Hellinger \& Travnicek (2008) and Sironi \& Narayan (2015) used an expanding/compressing box to accomplish the same goal of continually driving pressure anisotropy. Continuous driving of the pressure anisotropy is important because it allows one to study the nonlinear saturation of velocity-space instabilities, in contrast to more standard initial value calculations. One of the conclusions of the works of Kunz et al. (2014) and Riquelme et al. (2015) is that the mirror instability reaches nonlinear amplitudes with $\delta B \sim B$, independent of how slowly the magnetic field is amplified relative to the IC period $(\delta B \equiv|\delta \boldsymbol{B}|$ and $\delta \boldsymbol{B} \equiv \boldsymbol{B}-\langle\boldsymbol{B}\rangle ;$ throughout this paper, \langle\rangle will stand for the average of a quantity over the simulation volume or over a population of particles, depending on the quantity under consideration). A natural question is what effect these large amplitude mirrors have on the electron physics and how the whistler instability grows in the presence of large amplitude mirror modes.

In this paper we use particle-in-cell (PIC) simulations to study the combined effect of mirror and whistler instabilities on the electron pressure anisotropy. This work is thus an extension of our previous study of the ion-scale, kinetic instabilities (Riquelme et al. 2015). In order to properly separate phenomena occurring on the ion- and electron-lengthscales, 
in this work we use larger values of the ion to electron mass ratio: $m_{\mathrm{i}} / m_{\mathrm{e}}=64$ and 128 . We focus throughout this paper on a fiducial case with initial $\beta_{\mathrm{i}}=\beta_{\mathrm{e}}=20$ (in our simulations, $\beta_{j}$ decreases in time as the background magnetic field is amplified). At sufficiently low $\beta_{\mathrm{i}} \lesssim 1$, the IC instability is expected to be more important than the mirror instability in regulating the ion pressure anisotropy. However, Riquelme et al. (2015) did not find any significant differences in the nonlinear mirror evolution for $\beta_{\mathrm{i}}=20$ and $\beta_{\mathrm{i}}=80$, and even at $\beta_{\mathrm{i}}=6$ the IC instability was sub-dominant. Moreover, Kunz et al. (2014) found similar results at higher $\beta_{\mathrm{i}}=200$. Thus we believe that the calculations presented in this paper with $\beta_{\mathrm{i}}=20$ provide a good model for the saturation of electron velocity-space instabilities in $\beta_{\mathrm{i}} \gtrsim 1$ plasmas (at least for similar ion and electron temperatures, $T_{\mathrm{i}} \sim T_{\mathrm{e}}$; see Section 5).

Our work has two important applications. First, the nonlinear evolution of the mirror and whistler instabilities effectively sets the pitch-angle scattering rate (and thus the mean free path) of electrons in low collisionality plasmas. This in turn determines the viscosity and thermal conductivity of the plasma. In our calculations we will directly measure the electron mean free path $\left\langle\lambda_{\mathrm{e}}\right\rangle$ in the collisionless regime, and infer its physical dependence on the plasma parameters. Second, electron pressure anisotropy generates an "anisotropic viscosity" that can contribute to the heating of electrons in accretion disks and other low collisionality plasmas (Sharma et al. 2007). We will see that the magnitude of this heating depends on the magnitude of the electron pressure anisotropy produced by the combined effect of the whistler and mirror instabilities.

This paper is organized as follows. In Section 2 we describe the numerical set up of our runs, and our simulation strategy. In Section 3 we determine the saturated pressure anisotropy $\Delta p_{\mathrm{e}}$ for the electrons and quantify the electron heating due to anisotropic viscosity. In Section 4 we measure the mean free path of electrons and ions, and determine their dependence on the physical parameters of the plasma. In Section 5 we summarize our results and discuss their implications for galaxy clusters and low-collisionality black hole accretion flows.

\section{SIMULATION SETUP}

We use the electromagnetic, relativistic PIC code TRISTANMP (Buneman 1993, p. 67; Spitkovsky 2005) in two dimensions. The simulation box consists of a square box in the $x-y$ plane, containing plasma with a homogeneous initial magnetic field $\boldsymbol{B}_{0}=B_{0} \hat{x}$. Since we want to simulate a magnetic field that is being amplified in an incompressible way, we impose a velocity shear so that the mean particle velocity is $\boldsymbol{v}=-s x \hat{y}$, where $s$ is a shear parameter with units of frequency and $x$ is the distance along $\hat{x}$. From flux conservation, the $y$-component of the mean field evolves as $d\left\langle B_{y}\right\rangle / d t=-s B_{0}$. This implies a net growth of $|\langle\boldsymbol{B}\rangle|$, which in turn drives $p_{\perp, j}>p_{\|, j}$ during the whole simulation.

Simulations resolving the $x-y$ plane can capture mirror, IC, and whistler modes with wave vectors $\boldsymbol{k}$ forming any angle with the mean magnetic field $\langle\boldsymbol{B}\rangle$. In our previous study, focused on the interplay between the mirror and IC instabilities only (Riquelme et al. 2015), we found that most of the physics of the relevant instabilities is captured when the $x-y$ plane is resolved (as in the simulations in this paper).

The key parameters in our simulations are the particles' magnetization, quantified by the ratio between the initial cyclotron frequency of each species and the shear rate of the
Table 1

Physical and Numerical Parameters of the Simulations

\begin{tabular}{llccccr}
\hline \hline Runs & $m_{\mathrm{i}} / m_{\mathrm{e}}$ & $\omega_{c, \mathrm{e}} / s$ & $c / \omega_{p, \mathrm{e}} / \Delta_{x}$ & $N_{\mathrm{ppc}}$ & $L / R_{\mathrm{L}, \mathrm{i}}$ & $L / R_{\mathrm{L}, \mathrm{e}}$ \\
\hline OW1 & $\infty$ & 2500 & 5 & 60 & $\ldots$ & 48 \\
OW2 & $\infty$ & 1000 & 5 & 20 & $\ldots$ & 48 \\
MW1 & 64 & 2500 & 5 & 20 & 22 & 176 \\
MW2 & 64 & 2500 & 5 & 60 & 22 & 176 \\
MW3 & 128 & 5000 & 5 & 40 & 22 & 242 \\
\hline
\end{tabular}

Note. A summary of the physical and numerical parameters of the simulations discussed in the paper. These are the mass ratio $m_{\mathrm{i}} / m_{\mathrm{e}}$, the initial electron magnetization $\omega_{c, \mathrm{e}} / s$, the electron skin depth $c / \omega_{p, \mathrm{e}} / \Delta_{x}$ (where $\Delta_{x}$ is the grid point separation), the number of particles per cell $N_{\mathrm{ppc}}$ (including ions and electrons), the box size in units of the typical initial ion Larmor radius $L / R_{\mathrm{L}, \mathrm{i}}$ $\left(R_{\mathrm{L}, \mathrm{i}}=v_{\mathrm{th}, \mathrm{i}} / \omega_{c, \mathrm{i}}\right.$, where $v_{\mathrm{th}, \mathrm{i}}^{2}=k_{\mathrm{B}} T_{\mathrm{i}} / m_{\mathrm{i}}$ is the rms ion velocity and $k_{\mathrm{B}}$ and $T_{\mathrm{i}}$ are the Boltmann constant and the ion temperature, respectively), and the box size in terms of the typical initial electron Larmor radius $L / R_{\mathrm{L}, \mathrm{e}}$. We confirmed numerical convergence by varying resolution in $c / \omega_{c, \mathrm{e}} / \Delta_{x}, N_{\mathrm{ppc}}$, and $L / R_{\mathrm{L}, \mathrm{i}}$ $\left(L / R_{\mathrm{L}, \mathrm{e}}\right) . \quad$ All of the runs have $\beta_{\mathrm{i}}=\beta_{\mathrm{e}}=20, \quad k T_{\mathrm{e}} / m_{\mathrm{e}} c^{2}=0.28$, $\omega_{c, \mathrm{e}} / \omega_{p, \mathrm{e}}=0.17$, and $c=0.225 \Delta_{x} / \Delta_{t}$, where $\Delta_{t}$ is the simulation time step.

plasma, $\omega_{c, j} / s(j=i, e)$, and the ion to electron mass ratio, $m_{\mathrm{i}} / m_{\mathrm{e}}$. In typical astrophysical environments, $\omega_{c, j} \gg s$. Due to computational constraints, we will use values of $\omega_{c, j} / s \gg 1$, but still much smaller than expected in real astrophysical settings. Because of this, we have made sure to reach the regime where both $m_{\mathrm{i}} / m_{\mathrm{e}}$ and $\omega_{c, j} / s$ are large enough so that their values do not qualitatively affect any of our conclusions.

Our simulations have initial $\beta_{\mathrm{i}}=\beta_{\mathrm{e}}=20$. In all of our runs $k_{\mathrm{B}} T_{\mathrm{e}} / m_{\mathrm{e}} c^{2}=0.28$, which implies $\omega_{c, \mathrm{e}} / \omega_{p, \mathrm{e}}=0.17$ (where $k_{\mathrm{B}}$, $T_{\mathrm{e}}$, and $\omega_{p, \mathrm{e}}$ are the Boltzmann's constant, the electron temperature, and the electron plasma frequency). Thus the varying physical parameters in our simulations will be: $\omega_{c, \mathrm{e}} / s$ and $m_{\mathrm{i}} / m_{\mathrm{e}}$ (which uniquely fix $\omega_{c, \mathrm{i}} / s$ and $k_{B} T_{\mathrm{i}} / m_{\mathrm{i}} c^{2}$ ). Some of our simulations use "infinite mass ions" (the ions are technically immobile, so they just provide a neutralizing charge), with the goal of focusing on the electron-scale physics. These provide a useful contrast with our finite $m_{\mathrm{i}} / m_{\mathrm{e}}$ runs and allow us to isolate the impact of ion physics on the electrons. The numerical parameters in our simulations will be: $N_{\mathrm{ppc}}$ (number of particles per cell), $c / \omega_{p, \mathrm{e}} / \Delta_{x}$ (the electron skin depth in terms of grid size), $L / R_{\mathrm{L}, \mathrm{i}}$ (box size in terms of the initial ion Larmor radius for runs with finite $m_{\mathrm{i}} / m_{\mathrm{e}}$; $R_{\mathrm{L}, \mathrm{i}}=v_{\mathrm{th}, \mathrm{i}} / \omega_{c, \mathrm{i}}$, where $v_{\mathrm{th}, \mathrm{i}}^{2}=k_{\mathrm{B}} T_{\mathrm{i}} / m_{\mathrm{i}}$ is the rms ion velocity), and $L / R_{\mathrm{L}, \mathrm{e}}$ (box size in terms of the initial electron Larmor radius for runs with infinite $m_{\mathrm{i}} / m_{\mathrm{e}}$ ). Table 1 shows a summary of our key simulations. We ran a series of simulations ensuring that the numerical parameters (e.g., different $N_{\text {ppc }}$ ) do not significantly affect our results. Note that most runs used just for numerical convergence are not in Table 1.

\section{ELECTRON PHYSICS IN COLLISIONLESS SHEARING FLOWS}

In this section we quantify the nonlinear evolution of the electron pressure anisotropy in collisionless shearing flows, taking into account the combined effect of the nonlinear whistler and mirror instabilities. In order to understand the relative importance of the whistler and mirror modes, we start by isolating the effect of the whistler instability. We do this by using simulations where the ion mass is set to infinity, so that only the electron-scale whistler instability can grow. 

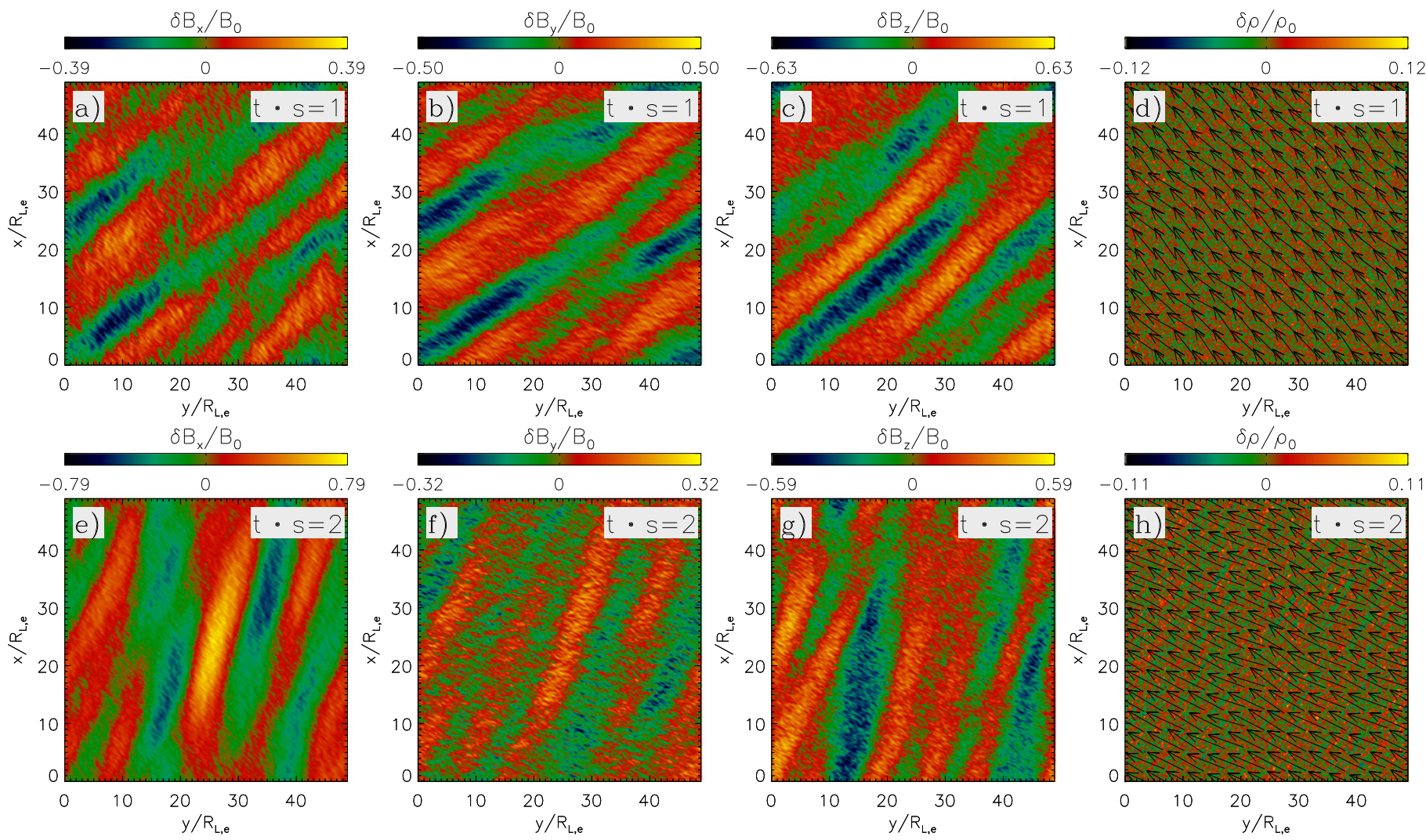

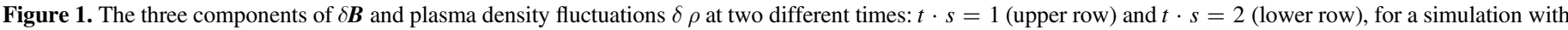

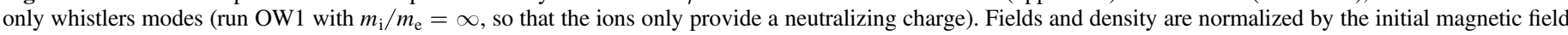

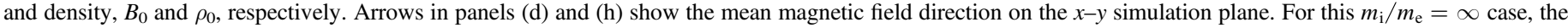
magnetic fluctuations are dominated by nearly parallel whistler modes.

\subsection{Simulations with Whistlers Only}

Figure 1 shows the magnetic field fluctuations and plasma density in a simulation in which the ions have an infinite mass and thus there is no mirror or IC instabilities (run OW1 in Table 1). In this simulation, the electrons are only affected by the whistler instability that develops when the electron pressure anisotropy increases due to the growth of the background magnetic field. The upper row in Figure 1 is at $t \cdot s=1$, i.e., after one shear time, while the lower row is at $t \cdot s=2$. Figure 1 shows that at all times the magnetic fluctuations are dominated by the nearly parallel whistler modes, with wavenumbers $k$ satisfying $k R_{\mathrm{L}, \mathrm{e}} \sim 0.5$; there are no ionlengthscale fluctuations contributing to $\delta \boldsymbol{B}$.

Figure 2 shows the initial evolution (until $t \cdot s=1$ ) of the electron pressures perpendicular (black-solid) and parallel (redsolid) to $\boldsymbol{B}$ for two runs, one with $\omega_{c, \mathrm{e}} / s=1000$ and one with $\omega_{c, \mathrm{e}} / s=2500$ (runs OW2 and OW1 in Table 1, respectively). The black- and red-dotted lines show the expectation from the CGL or double adiabatic limit (Chew et al. 1956), which is reasonably satisfied until $t \cdot s \sim 0.4$ in both simulations. After that, the growth of whistler modes provide enough pitch-angle scattering to break the adiabatic evolution of the electron pressure.

Figure 3 shows the time evolution of the magnetic fluctuations, the volume averaged pressure anisotropy, and the electron magnetic moment for the same runs until $t \cdot s=3$. Panels (c) and (d) show the volume averaged pressure anisotropy $\left\langle\Delta p_{\mathrm{e}}\right\rangle /\left\langle p_{\|, \mathrm{e}}\right\rangle$ for these two runs. For comparison, in both cases we plot the electron pressure anisotropy that would produce a whistler instability growth rate, $\gamma_{w}$, equal to five times the
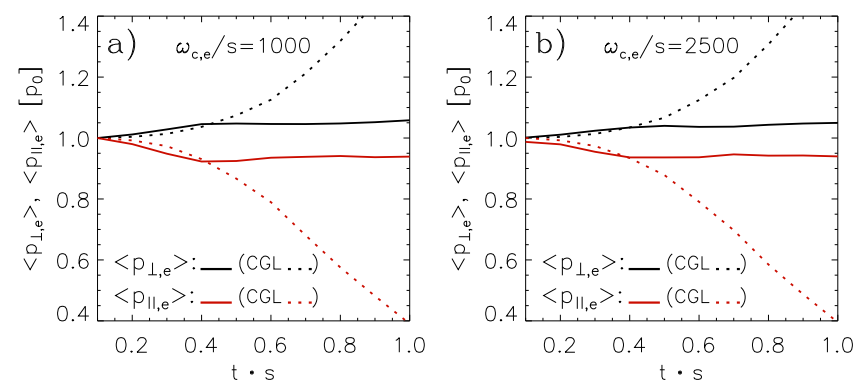

Figure 2. The initial evolution of the electron pressures perpendicular (blacksolid) and parallel (red-solid) to $\boldsymbol{B}$ for runs OW2 and OW1 in Table 1 (with $\omega_{c, \mathrm{e}} / s=1000$ and $\omega_{c, \mathrm{e}} / s=2500$, respectively). The black- and red-dotted lines show the expectation for the perpendicular and parallel pressures from the CGL or double adiabatic limit (Chew et al. 1956). Significant deviation from adiabatic evolution can be seen at $t \cdot s \gtrsim 0.4$.

shearing rate $\left(\gamma_{w}=5 s=5 \times 10^{-3} \omega_{c, \mathrm{e}} \quad\right.$ and $\quad \gamma_{w}=5 s=$ $2 \times 10^{-3} \omega_{c, \text { e }}$,respectively). These thresholds were obtained using the linear Vlasov solver developed by Verscharen et al. (2013) for mass ratio $m_{\mathrm{i}} / m_{\mathrm{e}}=1836 .{ }^{4}$ We see that in both cases there is

\footnotetext{
4 One subtlety is that the thresholds derived using the Vlasov solver of Verscharen et al. (2013) apply to the case of non-relativistic electrons. Since the electrons in our simulations are mildly relativistic $\left(k T_{\mathrm{e}}=0.28 m_{\mathrm{e}} c^{2}\right)$, we used initial-value PIC simulations to find a calibration factor $f_{\mathrm{c}}$ that scales the non-relativistic thresholds to the mildly relativistic regime (the threshold anisotropy for a given growth rate is larger by $f_{\mathrm{c}}$ in the mildly relativistic regime). For the whistler growth rates and mass ratios used in the paper, we found that $f_{\mathrm{c}}$ is a smooth function of $\beta_{\|, \mathrm{e}}$ only, with $f_{\mathrm{c}} \approx 1.5$ and 2 for $\beta_{\|, \mathrm{e}}=5$ and 15, respectively. Thus the whistler thresholds in Figure 3 and subsequent figures are the non-relativistic thresholds multiplied by $f_{\mathrm{c}}$.
} 

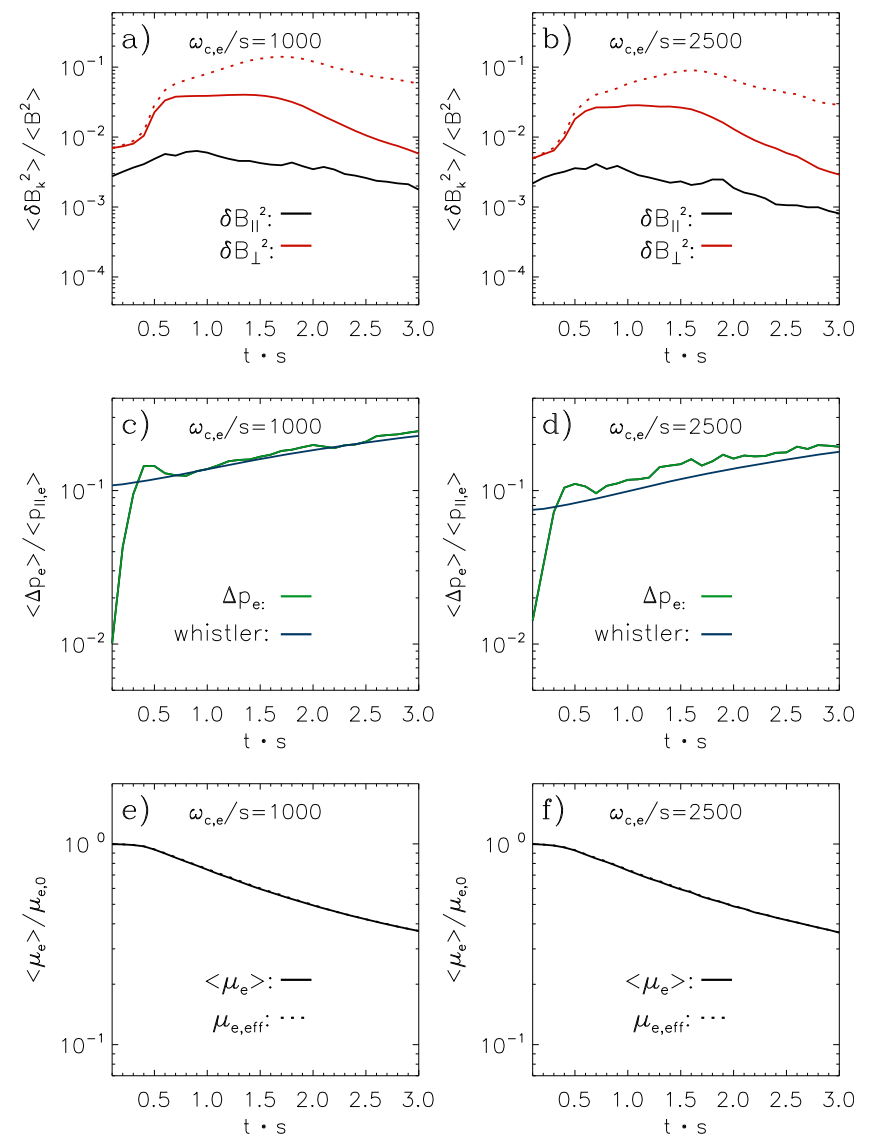

Figure 3. The evolution of different volume-averaged quantities for two simulations with $\omega_{c, \mathrm{e}} / s=1000$ (run OW2; left column) and $\omega_{c, \mathrm{e}} / s=2500$ (run OW1; right column), which use $m_{\mathrm{i}} / m_{\mathrm{e}}=\infty$ (the ions simply provide a neutralizing charge). Upper row: the volume-averaged magnetic energy components parallel and perpendicular to $\langle\boldsymbol{B}\rangle, \delta B_{\|}^{2}$ (black) and $\delta B_{\perp}^{2}$ (red), respectively (normalized by $\left\langle B^{2}\right\rangle$ ). For comparison, the red-dotted lines show $\delta B_{\perp}^{2}$ normalized by $B_{0}^{2}$. Middle row: the electron pressure anisotropy (green line), with the linear whistler instability thresholds for growth rates $\gamma_{w}=5 s$ (black line). The pressure anisotropy saturates at a value consistent with the linear instability threshold of the $\gamma_{w}=5 s$ modes. Lower row: the electron magnetic moment; see Equation (5) and associated discussion for definitions of $\mu_{\mathrm{e}}$ (solid) and $\mu_{\mathrm{e}, \mathrm{eff}}$ (dotted).

a reasonably good agreement between the electron anisotropy obtained from the simulation (using $m_{\mathrm{i}} / m_{\mathrm{e}}=\infty$ ) and the theoretical whistler instability thresholds. This shows that the electron anisotropy is maintained at roughly the marginal stability level for the whistler modes with $\gamma_{w}=5 s$. Note that in a realistic astrophysical or heliospherical environment, $\omega_{c, \mathrm{e}} / s$ is likely to be larger than in the simulations shown in Figure 3. This would lead to somewhat lower saturated $\Delta p_{\mathrm{e}} / p_{\|, \mathrm{e}}$ (see Section 3.2).

The pressure anisotropy evolution in Figure 3 is very similar for $\omega_{c, \mathrm{e}} / s=1000$ and $\omega_{c, \mathrm{e}} / s=2500$. The primary difference between these two runs is in the amplitude of $\delta \boldsymbol{B}$. This is shown in panels (a) and (b), where we plot the magnetic energy density in the components of $\delta \boldsymbol{B}$ that point along and perpendicular to $\langle\boldsymbol{B}\rangle$ (normalized by the average magnetic energy density in the simulation, $\left\langle B^{2}\right\rangle / 8 \pi$, which grows with time). We see first that $\delta \boldsymbol{B}$ is dominated by the component perpendicular to $\langle\boldsymbol{B}\rangle$, which is expected for the transverse nature of the whistler modes. Also, as the initial electron magnetization $\omega_{c, \mathrm{e}} / s$ increases by a factor 2.5 , the maximum value of $\delta \boldsymbol{B}^{2}$ decreases by a factor $\sim 1.5$. This behavior is consistent with the expectation that the whistler modes should produce an effective pitch angle scattering rate $\nu_{\text {eff }}$ proportional to $\omega_{c, \mathrm{e}}^{2}(\delta \boldsymbol{B} / B)^{2}$ (see, e.g., Marsch 2006). Indeed, under the assumption that $\nu_{\text {eff }}$ should maintain the electron pressure anisotropy at the marginally stable level for whistler growth (which happens for $t \cdot s \gtrsim 0.7$ in Figures 3(c) and (d)), the value of $\nu_{\text {eff }}$ can be estimated from the macroscopic properties of the flow as follows:

Let us consider the evolution of $p_{\perp, \mathrm{e}}$ and $U_{\mathrm{e}}=p_{\perp, \mathrm{e}}+p_{\|, \mathrm{e}} / 2$ in the case of an incompressible fluid, with homogeneous $p_{\perp, \mathrm{e}}$ and $p_{\|, \mathrm{e}}$, and without heat flux along $\langle\boldsymbol{B}\rangle$, which is given by (Kulsrud 1983; Snyder et al. 1997; Sharma et al. 2007):

$$
\frac{\partial p_{\perp, \mathrm{e}}}{\partial t}=-s p_{\perp, \mathrm{e}} B_{x} B_{y} / B^{2}-\frac{1}{3} \nu_{\mathrm{eff}}\left(p_{\perp, \mathrm{e}}-p_{\|, \mathrm{e}}\right)
$$

and

$$
\frac{\partial U_{\mathrm{e}}}{\partial t}=-s \Delta p_{\mathrm{e}} B_{x} B_{y} / B^{2} .
$$

If $\Delta p_{\mathrm{e}} / p_{\|, \mathrm{e}} \ll 1$ (which occurs in our case), $U_{\mathrm{e}} \approx 3 p_{\perp, \mathrm{e}} / 2$. Thus, assuming $B_{x} \sim\left|B_{y}\right| \sim B\left(B_{y}<0\right.$ in our case $)$, one gets that $\partial \ln \left(U_{\mathrm{e}}\right) / \partial t \sim \partial \ln \left(p_{\perp, \mathrm{e}}\right) / \partial t \sim s \Delta p_{\mathrm{e}} / p_{\|, \mathrm{e}} \ll s$. Equation (1) then implies that

$$
\nu_{\mathrm{eff}} \approx-3 s \frac{p_{\|, \mathrm{e}}}{\Delta p_{\mathrm{e}}} \frac{B_{x} B_{y}}{B^{2}} .
$$

Thus, comparing the $\nu_{\text {eff }}$ obtained in Equation (3) with the expected dependence of $\nu_{\text {eff }}$ on waves amplitude $\left(\nu_{\text {eff }} \propto \omega_{c, \mathrm{e}}^{2}\left(\delta B^{2} / B^{2}\right)\right)$ one obtains:

$$
\frac{\delta B^{2}}{B^{2}} \propto \frac{p_{\|, \mathrm{e}}}{\Delta p_{\mathrm{e}}} \frac{\left|B_{x} B_{y}\right|}{B^{2}} \frac{s}{\omega_{c, \mathrm{e}}^{2}} .
$$

Given that runs OW1 and OW2 have the same initial $\omega_{c, \mathrm{e}}$, the decrease in $s$ by a factor 2.5, along with the observed decrease in $\Delta p / p_{\|, \mathrm{e}}$ by a factor $\sim 1.5$ (see Figures 3(c) and (d)), is fairly consistent with the decrease in $\delta B^{2} / B^{2}$ by a factor $\sim 1.5$. Also, the roughly constant behavior of $\delta B^{2} / B^{2}$ in the range $t \cdot s \sim 0.5-1.5$ (see Figures $3(\mathrm{a})$ and (b)) is consistent with the fact that in the early stage of the saturated regime both $p_{\|, \mathrm{e}} / \Delta p_{\mathrm{e}}$ and $\omega_{c, \mathrm{e}}^{-2}$ decrease slowly with time, which is nearly compensated by the initial growth of $\left|B_{x} B_{y}\right| / B^{2}(\propto t)$. For $t \cdot s \gg 1$, the expectation is $\left|B_{x} B_{y}\right| / B^{2} \propto 1 / t$ and $\omega_{c, \mathrm{e}}^{-2} \propto 1 / t^{2}$, consistent with the rapid decrease of $\delta B^{2} / B^{2}$ at the end of the simulations. This behavior implies the absence of a long-term secular growth of $\delta B^{2} / B_{0}^{2}$, as can be seen from the red-dotted lines in Figures 3(a) and (b).

Finally, we define two magnetic moments to aid in interpreting the numerical results:

$$
\left\langle\mu_{j}\right\rangle \equiv\left\langle\frac{p_{\perp, j}}{B}\right\rangle \quad \text { and } \quad \mu_{j, \text { eff }} \equiv \frac{\left\langle p_{\perp, j}\right\rangle}{\langle B\rangle}
$$

$\left\langle\mu_{j}\right\rangle$ is the true volume averaged magnetic moment. In Riquelme et al. (2015) we showed that, in the case of the ions, $\mu_{i, \text { eff }} \neq\left\langle\mu_{\mathrm{i}}\right\rangle$, which is produced when there is a spatial correlation between $p_{\perp, i}$ and $B$, as in the case of large amplitude mirrors. Figures 3(e) and (f) compare these two definitions of the electron magnetic moment for the same runs OW2 and 
OW1. We see that for the two simulations, $\left\langle\mu_{\mathrm{e}}\right\rangle$ decreases on the same timescale $\left(\sim s^{-1}\right)$. The fact that $\left\langle\mu_{\mathrm{e}}\right\rangle$ and $\mu_{\mathrm{e}, \mathrm{eff}}$ are essentially indistinguishable in Figure 3 means that $p_{\perp, \mathrm{e}}$ does not fluctuate significantly in space. This is consistent with the relatively low amplitude fluctuations in $\delta B$ associated with the whistler instability. We will see below that this is no longer the case when mirror fluctuations are present.

\subsection{Simulations With Whistler and Mirror Modes}

In order to study the interplay between the electron-scale whistler instability and the ion-scale mirror instability, we now study a series of simulations with finite ion to electron mass ratios $m_{\mathrm{i}} / m_{\mathrm{e}}$. Ideally we would utilize $m_{\mathrm{i}} / m_{\mathrm{e}} \simeq 1836$ but this is infeasible given the need for both $2 \mathrm{D}$ and large ion and electron magnetization. Instead, we have tried to ensure that the simulations are in the regime where there is reasonable scale separation between ions and electrons. This is achieved for $m_{\mathrm{i}} /$ $m_{\mathrm{e}}=128$ but even at somewhat smaller mass ratios we find reasonably similar results.

As an example, Figure 4 shows the components of $\delta \boldsymbol{B}$ for run MW3 of Table $1\left(m_{\mathrm{i}} / m_{\mathrm{e}}=128\right.$ and $\left.\omega_{c, \mathrm{e}} / s=5000\right)$. The upper and lower rows correspond to $t \cdot s=1$ and $t \cdot s=2$, respectively. At $t \cdot s=1$ the oblique mirror modes are visible in $\delta B_{x}$ and $\delta B_{y}$, while the whistler modes are most clearly seen in Figure 4(c), which shows $\delta B_{z}$. At $t \cdot s=2$ we see a well developed highly nonlinear stage of all the instabilities. Whereas $\delta \boldsymbol{B}$ is dominated by the mirror modes (with wavenumber $k$ such that $k R_{\mathrm{L}, \mathrm{i}} \sim 1$, where $R_{\mathrm{L}, \mathrm{i}}$ is the ion Larmor radius), the $\delta B_{z}$ component also shows the (subdominant) presence of the IC modes. This is consistent with our previous results (Riquelme et al. 2015): although subdominant, the IC instability persists for $\beta_{\mathrm{i}} \sim 10$ (though it is not present at higher $\beta_{\mathrm{i}}$ ). Figure 4(h) also shows significant plasma density fluctuations, which correlate well with the mirror modes. At $t \cdot s=2$, the three components of $\delta \boldsymbol{B}$ also show the presence of parallel whistler modes on scales comparable to $R_{\mathrm{L}, \mathrm{e}} \approx R_{\mathrm{L}, \mathrm{i}} / 11$ (consistent with $\sqrt{m_{\mathrm{i}} / m_{\mathrm{e}}} \approx 11$ ).

The presence of the different ion- and electron-scale modes can also be seen from Figure 5. Figures 5(a) and (b) show the Fourier transform of $\delta B_{z}$ at $t \cdot s=1$ and $t \cdot s=2$, respectively, as a function of the wavenumbers parallel and perpendicular to $\langle\boldsymbol{B}\rangle$. At $t \cdot s=1$, the quasi-parallel whistler modes with $k R_{\mathrm{L}, \mathrm{e}} \sim 0.5$ contribute most of the power, with the contribution of smaller wavenumber, quasi-parallel and oblique modes (IC and mirror modes, respectively) being subdominant. At $t \cdot s=2$, the whistler, IC, and mirror modes continue to contribute $B_{z}$ fluctuations in similar regions of the $k_{\|}-k_{\perp}$ space, but with the whistler modes having significantly less power compared to the IC and mirror modes. Figure 5(c) shows $B_{x}$ and $B_{z}$ fluctuations in a small one-dimensional region (marked by a small black line in Figure 4(f)) at $t \cdot s=2$. The presence of whistler modes with $k R_{\mathrm{L}, \mathrm{e}} \sim 0.5$ appears clearly as low amplitude fluctuations (relative to the mirror modes).

Figure 6 compares the evolution of the energy in $\delta \boldsymbol{B}$, the ion and electron anisotropies, and $\mu_{\mathrm{i}}$ and $\mu_{\mathrm{e}}$ for simulations with $m_{\mathrm{i}} / m_{\mathrm{e}}=64$ and 128 (runs MW2 and MW3 in Table 1), and demonstrates that the physics of electron isotropization is fairly well converged for these two mass ratios. Although these two simulations differ in $m_{\mathrm{i}} / m_{\mathrm{e}}$, the ions are under the same conditions $\left(\omega_{c, \mathrm{i}} / s=40\right.$ and $\beta_{i}=20$ in the two cases). The electrons are also under similar conditions (the same $\beta_{e}=20$,
$k_{\mathrm{B}} T_{\mathrm{e}}=0.28 m_{\mathrm{e}} c^{2}$ ), but their magnetizations differ by a factor 2 (see Table 1), which is required by the factor 2 difference in $m_{\mathrm{i}} / m_{\mathrm{e}}$. Figures 6(a) and (b) show the magnitude of the volumeaveraged magnetic energy in fluctuations parallel and perpendicular to the volume-averaged (shearing) magnetic field, $\langle\boldsymbol{B}\rangle(t)$, normalized by $\left\langle B^{2}\right\rangle$. We see that, for both mass ratios, the amplitude of the mirror modes is about the same at saturation, confirming the results of Kunz et al. (2014) and Riquelme et al. (2015) that $\delta B / B \sim 0.3$ in the saturated mirror state. There is also a subdominant perpendicular field component, $\delta B_{\perp}^{2}$, which is at first dominated by the whistler modes (most clearly seen by the exponential growth at $t \cdot s \approx 0.5$ in Figures 6(a) and (b)), and then by the IC modes at later times.

Figures 6(c) and (d) show the volume-averaged electron and ion pressure anisotropies as a function of time (green and black lines, respectively). The anisotropy evolution for the two species is essentially the same for the two mass ratios, although there are small quantitative differences. Figures 6(c) and (d) also show the anisotropy threshold for mirror (red line) and whistler modes (blue line). The linear theory mirror instability threshold for growth rate equal to $s$ reasonably describes the saturation of the ion pressure anisotropy (aside from the overshoot at $t \cdot s \sim 1$ that is unavoidable at the modest ratios of the IC frequency to the shear rate used here). However, the electron pressure anisotropy is a factor $\sim 1.5-2$ smaller than the linear theory threshold for whistler modes. ${ }^{5}$ This suggests that the factor $\sim 1.5-2$ decrease in $\Delta p_{\mathrm{e}} / p_{\|, \mathrm{e}}$ is caused by the presence of the mirror modes in the simulations with finite mass ratios (runs MW2 and MW3).

This reduction in $\Delta p_{\mathrm{e}} / p_{\|, \mathrm{e}}$ can be understood in terms of the effect of the nonlinear mirror modes on the electrons. The mirrors tend to bunch electrons (and ions) into low magnetic energy regions, which contributes to reducing the volumeaveraged anisotropy. This can also be seen from panels 6(e) and (f), where we compare $\left\langle\mu_{j}\right\rangle=\left\langle p_{\perp, j} / B\right\rangle$ and $\mu_{j, \text { eff }}=\left\langle p_{\perp, j}\right\rangle /\langle B\rangle$ for both ions and electrons. We see that $\mu_{j \text {,eff }}$ tends to be noticeably smaller than $\left\langle\mu_{j}\right\rangle$ (by $\sim 20 \%$ ) at $t \cdot s \gtrsim 1$, implying that the mirror modes partially reduce $p_{\perp, j}$ in a way that conserves $\mu_{j}$ (bunching them into mirrors).

This effect can also be seen from Figure 7, which compares the spatial distributions of $\Delta p_{\mathrm{e}} / p_{\|, \mathrm{e}}$ (Figure 7(a)) and $B^{2}$ (Figure 7(b)) for run MW3 at $t \cdot s=2$ (the same run and time shown in the lower row of Figure 4). The presence of significant $\Delta p_{\mathrm{e}} / p_{\|, \mathrm{e}}$ fluctuations on scales comparable to the typical scales of the (mirror-dominated) $B^{2}$ variations underscores the importance of the mirror modes in regulating $\Delta p_{\mathrm{e}} / p_{\|, \mathrm{e}}$. The effect of mirror modes on $\left\langle\Delta p_{\mathrm{e}}\right\rangle /\left\langle p_{\|, \mathrm{e}}\right\rangle$ in the $m_{\mathrm{i}} /$ $m_{\mathrm{e}}=128$ case, however, is smaller than in the $m_{\mathrm{i}} / m_{\mathrm{e}}=64$ case, suggesting that the mirrors would have less of an effect on the electron anisotropy (relative to the whistler modes) at even larger $m_{\mathrm{i}} / m_{\mathrm{e}}$. We thus consider the factor $\sim 1.5-2$ an upper limit for the effect of mirror modes on the electron anisotropy.

One noticeable difference between the ion and electron response to the growing magnetic field is that $\mu_{\mathrm{i}}$ is conserved to reasonably high accuracy for $t \cdot s \lesssim 1$, while $\mu_{\mathrm{e}}$ is not (Figures 6(e) and (f)). This is because the mirror instability has a secular phase that conserves $\mu_{\mathrm{i}}$ (Schekochihin et al. 2005; Kunz et al. 2014). The adiabatic invariance of $\mu_{\mathrm{i}}$ is only broken

\footnotetext{
5 In the case of whistler modes, the theoretical thresholds correspond to growth rates of $5 s$, since these are the rates that fit fairly well the electron anisotropies in the case of "infinite mass" ions (see Figures 3(c) and (d)).
} 

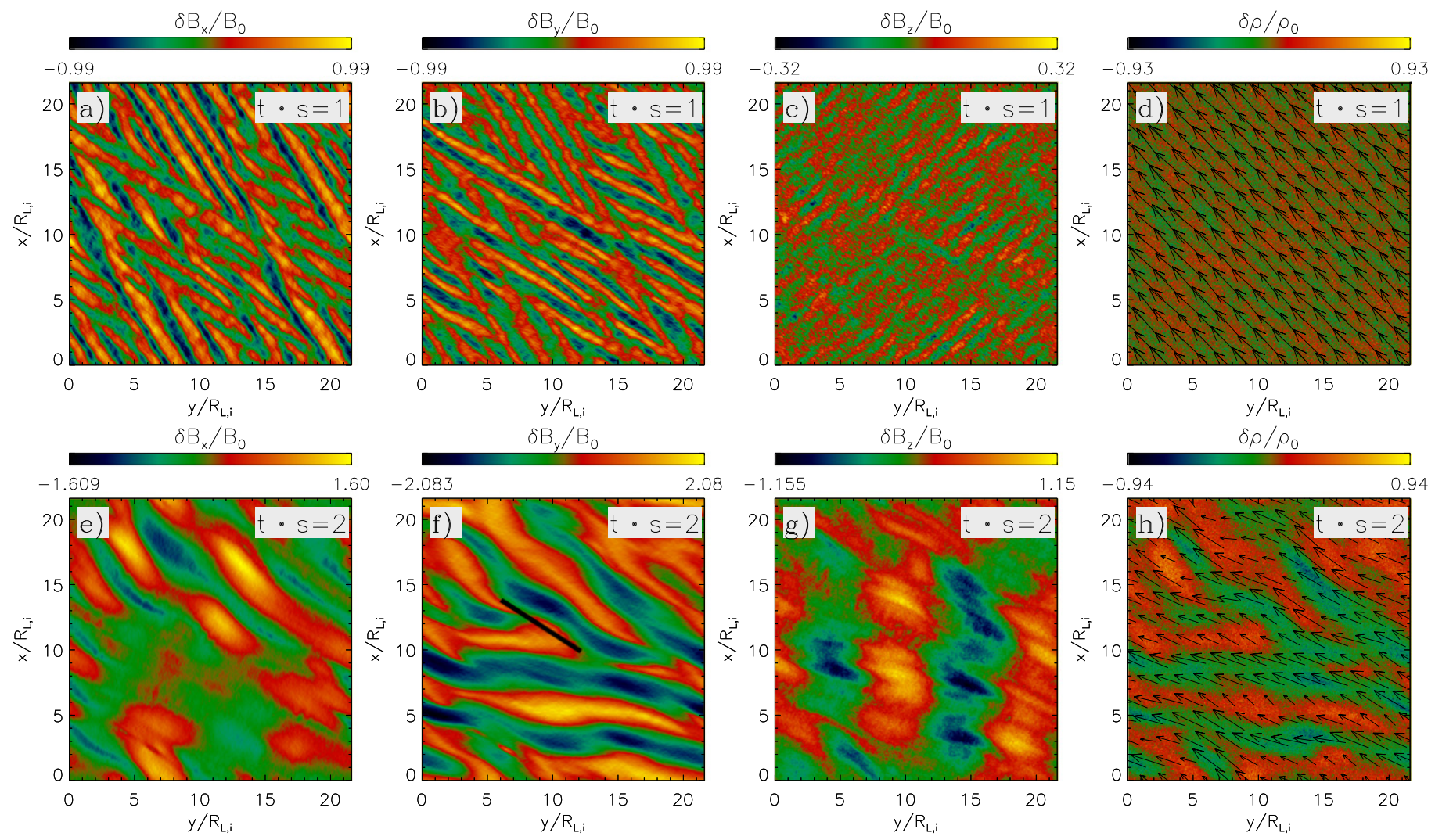

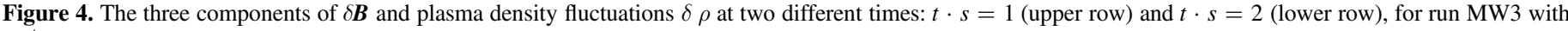

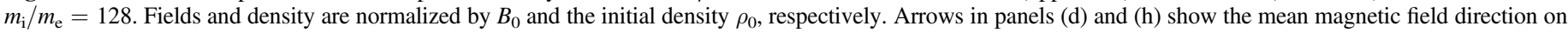

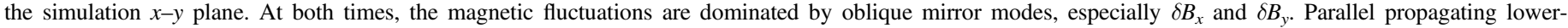

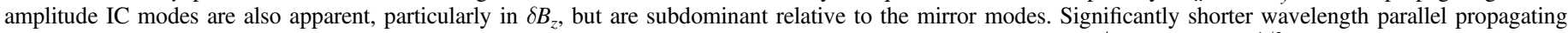

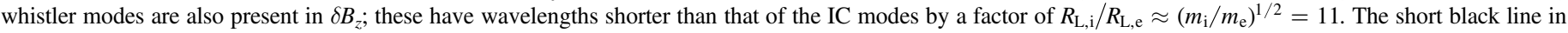
panel (f) shows a small region where the whistler modes are apparent. The corresponding fluctuations are shown in Figure 5(b).
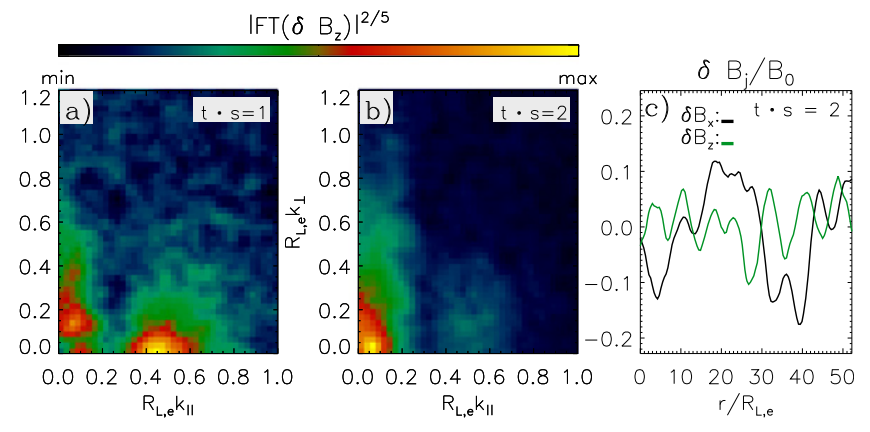

Figure 5. The signatures of whistler modes for run MW3. Plot a: the magnitude of the Fourier transform of $\delta B_{z}$ at $t \cdot s=1$ (raised to the $2 / 5$ power, $\left|\mathrm{FT}\left(\delta B_{z}\right)\right|^{2 / 5}$, to provide better dynamical range), as a function of the wavenumbers parallel and perpendicular to $\langle\boldsymbol{B}\rangle\left(k_{\|}\right.$and $k_{\perp}$, respectively). The contribution from quasi-parallel whistler modes with wavevectors satisfying $k R_{\mathrm{L}, \mathrm{e}} \sim 0.5$, along with the subdominant contributions of the longer wavelength IC and mirror modes (quasi-parallel and oblique, respectively) are clearly seen. Plot b: same as in plot a, but at $t \cdot s=2$. In this case, most of the power is provided by the IC and mirror modes, with the whistler modes contributing subdominant power. Plot c: the $\delta B_{x}$ and $\delta B_{z}$ components of $\delta \boldsymbol{B}$ in a small onedimensional region of the run at $t \cdot s=2$, revealing the presence of whistler modes with $k R_{\mathrm{L}, \mathrm{e}} \sim 0.5$. The location of the $1 \mathrm{D}$ region of this plot in the larger 2D computational plane is shown with the short black line in Figure 4(f).

when the mirrors reach $\delta B \sim B$, which happens at $t \cdot s \sim 1$. By contrast, the electron magnetic moment decreases at much earlier times. This is due to the electron whistler instability which does not have a secular phase and which can pitch angle scatter the electrons at low amplitudes, and hence at $t \cdot s \lesssim 1$.

\subsection{Viscous Heating}

Figure 6 demonstrates the existence of a quasi-steady state pressure anisotropy at a level set by the threshold of the mirror/ whistler instabilities. This in turn corresponds to an effective viscosity for both ions and electrons: in the present context, $P_{x y} \propto\left(p_{\perp}-p_{\|}\right) B_{x} B_{y} / B^{2}$ since the particles are roughly gyrotropic in velocity-space, where $P_{x y}$ is the $x-y$ component of the pressure tensor. In our simulations, this anisotropic pressure can tap into the velocity shear in the plasma, converting shear energy into random thermal energy. To quantify the importance of this heating mechanism in our simulations, Figure 8(a) shows the volume-averaged ion (solid-black) and electron (solid-geen) heating rates for run MW2: $d\left\langle U_{j}\right\rangle / d t$, where $U_{j}$ is the internal energy per unit volume of species $j$. For comparison, we also plot the expected ion (dotted-black) and electron (dotted-green) heating rates due to the work done by anisotropic viscosity: $-s\left\langle\Delta p_{j} B_{x} B_{y} / B^{2}\right\rangle$, which is obtained from Equation (2). ${ }^{6}$

For both for ions and electrons there is good agreement between the particle heating in the simulation and the contribution from the anisotropic stress. In the case of the electrons the measured heating is moderately larger (by a factor

\footnotetext{
6 We compared the heating predicted by two different volume averages: $-s\left\langle\Delta p B_{x} B_{y} / B^{2}\right\rangle$ and $-s\langle\Delta p\rangle\left\langle B_{x}\right\rangle\left\langle B_{y}\right\rangle /\left\langle B^{2}\right\rangle$. The results were nearly indistinguishable at all times. This implies that the correlations in the fluctuating fields do not significantly change the heating rate in these calculations, even in the presence of large amplitude mirrors.
} 

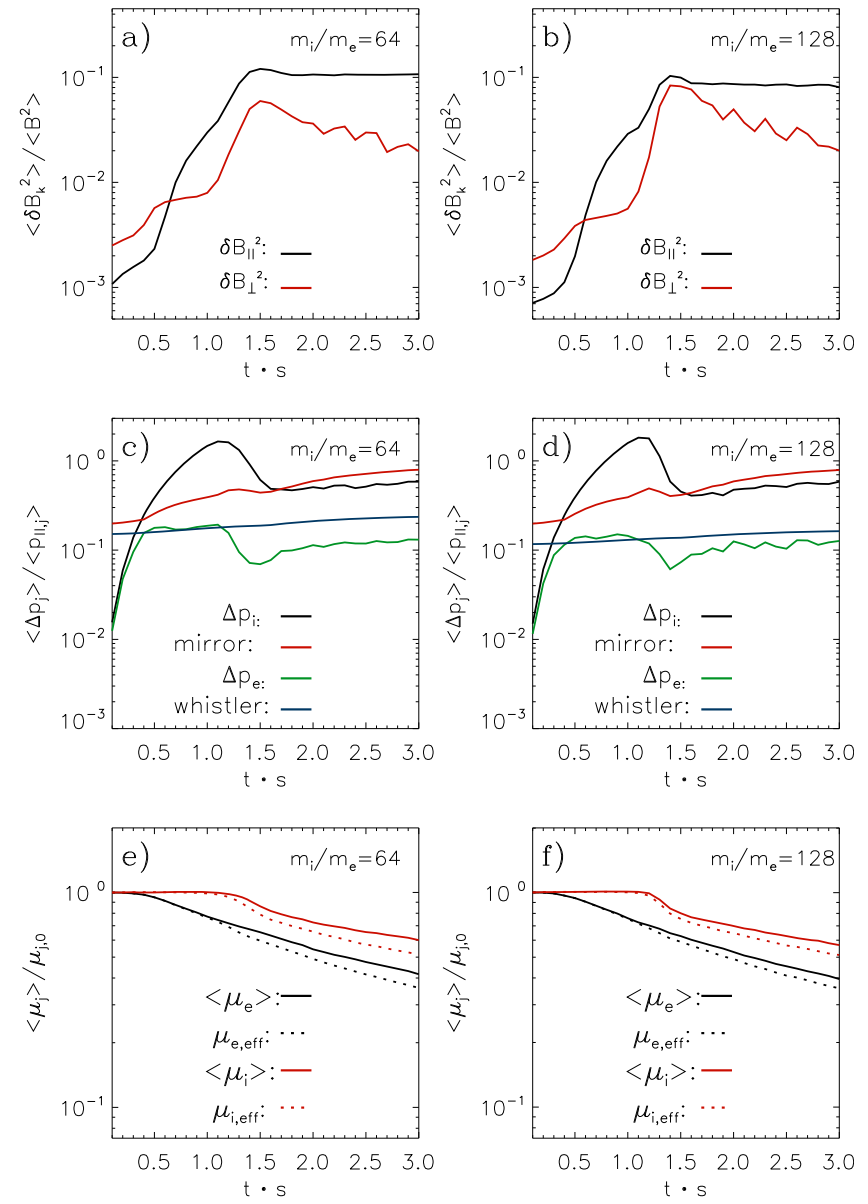

Figure 6. Time evolution of volume-averaged properties for simulations with $m_{\mathrm{i}} / m_{\mathrm{e}}=64$ (left column; run MW2) and $m_{\mathrm{i}} / m_{\mathrm{e}}=128$ (right column; run MW3). The upper row shows the evolution of the magnetic energy parallel (black) and perpendicular (red) to $\langle\boldsymbol{B}\rangle$, normalized by $B^{2} / 8 \pi$. The middle row shows the ion (black) and electron (green) pressure anisotropies, $\Delta p_{j} / p_{\|, j}$. Panels (c) and (d) also contain the anisotropy thresholds for mirror (red) and whistler (blue) modes growing at growth rates of $\sim s$ (using $m_{\mathrm{i}} / m_{\mathrm{e}}=64$ and 128 , respectively). The electron pressure anisotropy saturates at a value $\sim 2$ times lower than the value expected if the isotropization were dominated by whistler modes only; this is due to bunching by the large-amplitude mirrors generated by the ions. The lower row shows the ion (red) and electron (black) magnetic moments, defined as in Equation (5), and normalized by the initial value of $\mu_{j}$.
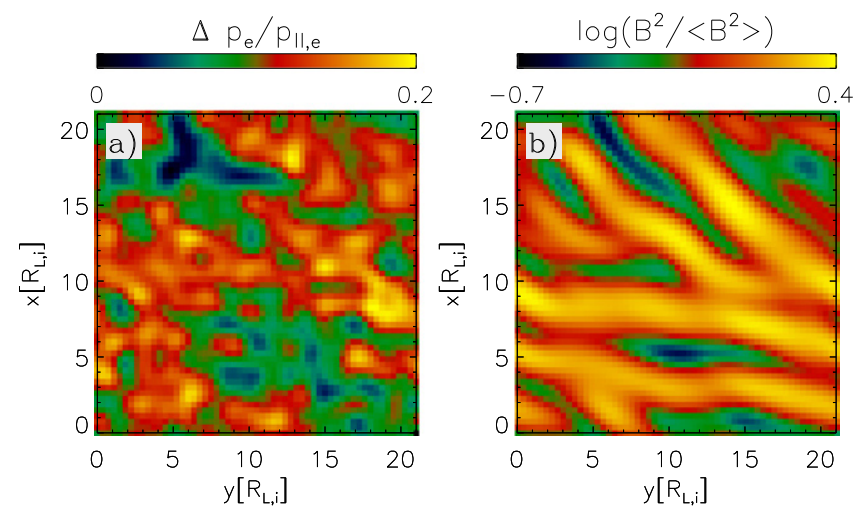

Figure 7. Comparison of the spatial distributions of $\Delta p_{\mathrm{e}} / p_{\|, \mathrm{e}}$ (panel (a)) and $B^{2}$ (panel (b)) for run MW3 at $t \cdot s=2$ (same run and time shown in the lower row of Figure 4).

1.5) than the viscous heating expectation. This can be seen more clearly in Figure 8(b), which shows the ratio between the measured electron heating and the expected contribution from
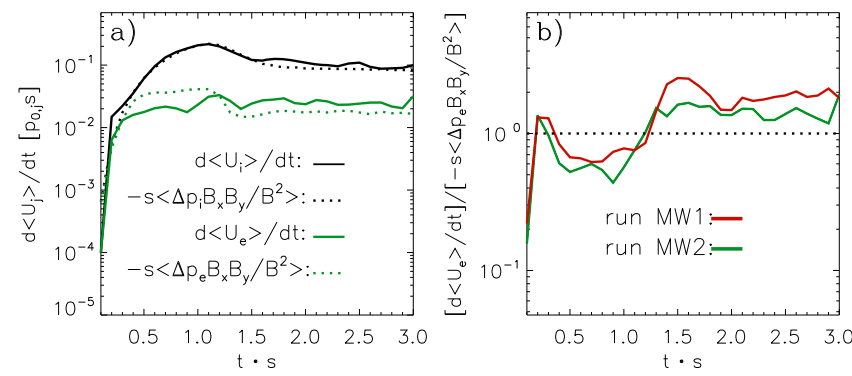

Figure 8. Panel (a): the ion and electron heating rates for run MW2, calculated directly via $d\left\langle U_{\mathrm{i}}\right\rangle / d t$ and $d\left\langle U_{\mathrm{e}}\right\rangle / d t$ (solid black and green, respectively), where $U_{j}$ is the internal energy per unit volume of species $j$. For comparison, we also show that the numerically calculated heating rates are well explained by the theoretically predicted ion (dotted-black) and electron (dotted-green) energy gain via "anisotropic viscosity" tapping into the background velocity shear (see Equation (2)). Panel (b): the ratio between the electron heating, $d\left\langle U_{\mathrm{e}}\right\rangle / d t$, and the expectation from viscous heating for runs MW1 (red) and MW2 (green), which only differ in their $N_{\text {ppc }}$ (=20 and 60, respectively).

anisotropic viscosity for run MW2 (green line). For comparison, we also show the case of run MW1 (red line), which uses $N_{\mathrm{ppc}}=20$ (instead of $N_{\mathrm{ppc}}=60$, as in run MW2; all the other parameters are the same). The fact that run MW1 shows an extra factor $\sim 1.5$ increase in the electron heating suggests that a significant contribution to the additional heating comes from the numerical noise due to the limited values of $N_{\mathrm{ppc}}$ feasible in our simulations.

It has also been argued that the energy transfer from ionscale turbulent fluctuations to the electrons could be a possible source of electron heating (Sironi \& Narayan 2015, though in a regime where the IC instability dominates over the mirror instability; see Section 5). Another possible factor at play is that the electrons' energy could be somewhat reduced by the growth of the waves' energy itself. This effect appears to be significant in the early part of the simulations, when the energy content in the mirror/IC/whistler fluctuations is rapidly growing. Indeed, Figure 8 shows that for $t \cdot s \lesssim 1.2$ the heating rate of the electrons is somewhat smaller than the viscous heating prediction. However, despite these considerations, the electron heating obtained for run MW2 shows that the anisotropic viscosity constitutes the dominant mechanism for electron heating in our simulations.

\section{ELECTRON MEAN FREE PATH}

The pitch-angle scattering created by velocity-space instabilities provides an upper limit to the particles' mean free path in a low collisionality plasma. This in turn determines the magnitude of the effective viscosity and thermal conductivity in the plasma. In this section we quantify this directly by computing the ion and electron mean free paths $\left(\lambda_{\mathrm{i}}\right.$ and $\left.\lambda_{e}\right)$ along the mean magnetic field, $\langle\boldsymbol{B}\rangle$, during the nonlinear stage of the whistler and mirror instabilities. In order to do so, in each simulation we compute the distance $D_{j}(t)$ traveled along $\langle\boldsymbol{B}\rangle$ for

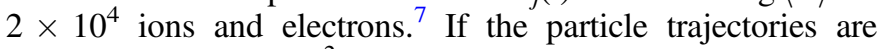
random walks, then $\left\langle D_{j}^{2}\right\rangle=t v_{\mathrm{th}, j}\left\langle\lambda_{j}\right\rangle$ (where $\left\langle\lambda_{j}\right\rangle$ represents the average mean free path over species $j$, and $v_{\mathrm{th}, j}=\left(k_{\mathrm{B}} T_{j} / m_{j}\right)^{1 / 2}$ is the thermal speed). Calculating $d\left\langle D_{j}^{2}\right\rangle / d t$ then gives an estimate of the average mean free path $\left\langle\lambda_{j}\right\rangle$ of species $j$. As in Section 3, we first describe our calculation of $\left\langle\lambda_{\mathrm{e}}\right\rangle$ for

\footnotetext{
$D_{j}(t) \equiv \int_{0}^{t} \boldsymbol{v}_{j} \cdot \boldsymbol{B} / B d t$, where $\boldsymbol{v}_{j}$ is the particle's velocity.
} 


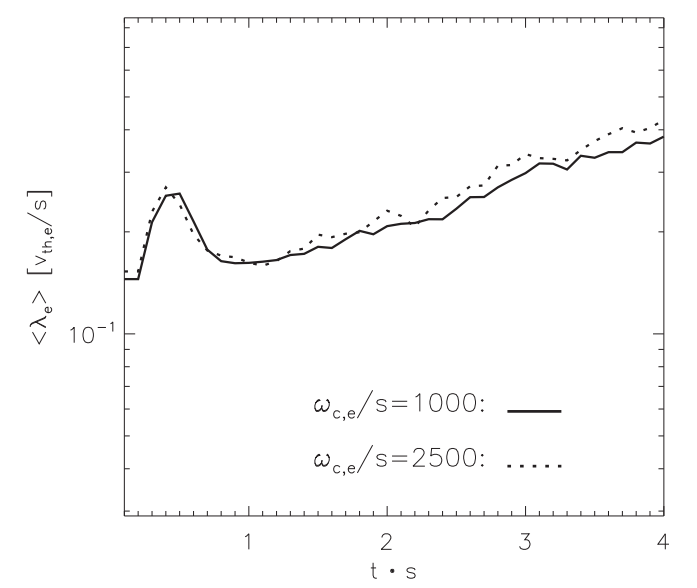

Figure 9. The average electron mean free path, $\left\langle\lambda_{\mathrm{e}}\right\rangle$, normalized by $v_{\text {th,e }} / s$ and calculated via the time derivative of the mean squared distance traveled by electrons along the mean magnetic field $\langle\boldsymbol{B}\rangle$, for runs with infinite ion mass and with $\omega_{c, \mathrm{e}} / s=1000$ (solid; run OW2) and $\omega_{c, \mathrm{e}} / s=2500$ (dotted; run OW1). There is no significant dependence of the estimated mean free path on the magnetization $\omega_{c, \mathrm{e}} / s$. Aside from an early free streaming phase, the mean free path is well estimated via $\left\langle\lambda_{\mathrm{e}}\right\rangle \approx 0.3\left(\left\langle\Delta p_{\mathrm{e}}\right\rangle /\left\langle p_{\|, \mathrm{e}}\right\rangle\right)\left(B^{2} /\left|B_{x} B_{y}\right|\right) v_{\text {th,e }} / s$ (see Equations (1) and (2) and associated discussion). The late-time increase in the electron mean free path is consistent with the increase in $\left\langle\Delta p_{\mathrm{e}}\right\rangle /\left\langle p_{\|, \mathrm{e}}\right\rangle$ and $B^{2} /\left|B_{x} B_{y}\right|$.

simulations with infinite mass ions. This way we will clearly separate the effect of mirror and whistler modes on $\left\langle\lambda_{\mathrm{e}}\right\rangle$.

Figure 9 shows $\left\langle\lambda_{\mathrm{e}}\right\rangle \equiv d\left\langle D_{\mathrm{e}}^{2}\right\rangle / d t / v_{\text {th,e }}$ (normalized by $v_{\text {th,e }} / s$ ) for simulations with infinite mass ions and for electron magnetizations, $\omega_{c, \mathrm{e}} / s=1000$ and 2500 (simulations OW2 and OW1, respectively). The evolution of $\left\langle\lambda_{\mathrm{e}}\right\rangle$ for the two electron magnetizations is very similar. At the beginning there is a small period of time when $\left\langle\lambda_{\mathrm{e}}\right\rangle$ increases rapidly $\sim t$. This is consistent with an initial "free streaming" of the electrons (in which $\left.d\left\langle D_{\mathrm{e}}^{2}\right\rangle / d t \propto t\right)$, followed by a sudden decrease in the mean free path due to the strong scattering at the end of the exponential whistler growth phase (where a transient anisotropy "overshoot" occurs, leading to an overshoot in the mean free path; see Figures 3(c) and (d), corresponding to the same runs OW2 and OW1). By $t \cdot s \sim 0.5$, the whistler modes have reached the fully saturated regime, and $\left\langle\lambda_{\mathrm{e}}\right\rangle \sim 0.15 v_{\text {th,e }} / s$. After that, $\left\langle\lambda_{\mathrm{e}}\right\rangle$ grows with time, increasing by a factor of $\sim 2$ by the end of the simulation.

The numerically determined evolution of $\left\langle\lambda_{\mathrm{e}}\right\rangle$ can be understood by considering the expression for $\nu_{\text {eff }}$ given by Equation (3), which is equivalent to $\left\langle\lambda_{\mathrm{e}}\right\rangle \approx 0.3\left(\left\langle\Delta p_{\mathrm{e}}\right\rangle /\left\langle p_{\|, \mathrm{e}}\right\rangle\right)\left(B^{2} /\left|B_{x} B_{y}\right|\right) v_{\text {th, }} / s$. At $t \cdot s=1$, the simulations with fixed ions have $\left\langle\Delta p_{\mathrm{e}}\right\rangle /\left\langle p_{\|, \mathrm{e}}\right\rangle \simeq 0.15$ (Figures 3(c) and (d)) and $B^{2} /\left|B_{x} B_{y}\right| \simeq 2$, which corresponds to $\left\langle\lambda_{\mathrm{e}}\right\rangle \approx 0.15 v_{\text {th,e }} / s$. This is in reasonable agreement with the numerically determined values in Figure 9. The factor $\sim 2$ increase in the electron mean free path from $t \cdot s=1$ to $t \cdot s=3$ in Figure 9 is consistent with the factor $\sim 1.5$ increase in both $\Delta p_{\mathrm{e}} / p_{\|, \mathrm{e}}$ (due to the decreasing $\beta_{\mathrm{e}}$, which increases the threshold pressure anisotropy for the whistler instability) and in $B^{2} /\left|B_{x} B_{y}\right|$ (from $\sim 2$ to $\sim 3$ ).

Figure 10 shows our calculations of the electron and ion mean free paths for simulations with $m_{\mathrm{i}} / m_{\mathrm{e}}=64$ and 128 (runs MW2 and MW3, respectively). Relatively independent of the mass ratio, the net effect of the mirror modes is to reduce the electron mean free path by a factor $\sim 2$ relative to the whistler-only results in Figure 9 . Since the mirror modes also reduce $\left\langle\Delta p_{\mathrm{e}}\right\rangle /\left\langle p_{\|, \mathrm{e}}\right\rangle$ by a factor of $\sim 1.5-2$, the result

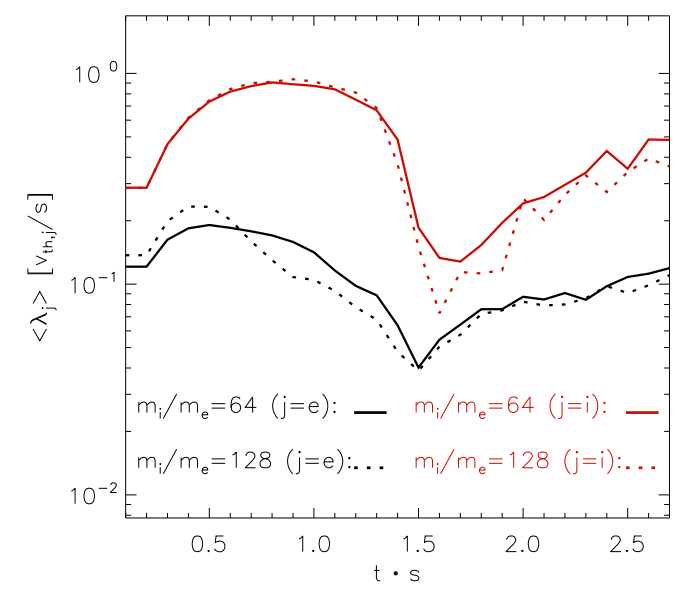

Figure 10. Electron (black) and ion (red) mean free paths (normalized by $v_{\mathrm{th}, j} / s$ ), calculated via the time derivative of the mean squared distance traveled by particles along $\langle\boldsymbol{B}\rangle$. We show results for runs with $m_{\mathrm{i}} / m_{\mathrm{e}}=64$ (solid lines; run $\mathrm{MW} 2$ ) and $m_{\mathrm{i}} / m_{\mathrm{e}}=128$ (dotted lines; run MW3). At early times the particles undergo a period of free-streaming in which the inferred mean free path increases. After the velocity-space instabilities saturate, however, pitch angle scattering ensues leading to a rough saturation of the mean free path. The simulations with different mass ratios give similar results, with $\left\langle\lambda_{j}\right\rangle \approx 0.3\left(\left\langle\Delta p_{j}\right\rangle /\left\langle p_{\|, j}\right\rangle\right)\left(B^{2} /\left|B_{x} B_{y}\right|\right) v_{\text {th }} / s$ in both cases (see Equations (1) and (2) and associated discussion).

$\left\langle\lambda_{\mathrm{e}}\right\rangle \approx 0.3\left(\left\langle\Delta p_{\mathrm{e}}\right\rangle /\left\langle p_{\|, \mathrm{e}}\right\rangle\right)\left(B^{2} /\left|B_{x} B_{y}\right|\right) v_{\text {th, } \mathrm{e}} / s$ derived above continues to describe the behavior of $\left\langle\lambda_{\mathrm{e}}\right\rangle$ when mirror modes are present. Figure 10 also shows the inferred average ion mean free path, $\left\langle\lambda_{i}\right\rangle s / v_{\text {th,i }}$, which is a factor of $\sim 3$ larger than that of the electrons. This is consistent with both species satisfying $\left\langle\lambda_{j}\right\rangle \approx 0.3\left(\left\langle\Delta p_{j}\right\rangle /\left\langle p_{\|, j}\right\rangle\right)\left(B^{2} /\left|B_{x} B_{y}\right|\right) v_{\text {th, }, j} / s$ given that $\left\langle\Delta p_{\mathrm{i}}\right\rangle /\left\langle p_{\|, i}\right\rangle$ is a factor $\sim 3$ larger than $\left\langle\Delta p_{\mathrm{e}}\right\rangle /\left\langle p_{\|, \mathrm{e}}\right\rangle$ in our simulations (see Figures 6(c) and (d)).

Figure 11 compares the probability distributions of mean free paths, $\lambda_{\mathrm{e}}$, for electrons in runs OW1 (only whistlers; green line) and MW1 (whistlers and mirrors; black line). This is done by measuring the distance $d$ traveled by each electron along $\boldsymbol{B}$ during an interval $\Delta t=s^{-1}$, from $t \cdot s=1.5$ to 2.5. This allows to estimate $\lambda_{\mathrm{e}}$ for each individual electron by assuming $d^{2}=\lambda_{\mathrm{e}} v_{\text {th,e }} \Delta t .^{8}$ The vertical-dotted green and black lines mark the average $\left\langle\lambda_{\mathrm{e}}\right\rangle$ for the OW1 and MW1 runs, respectively, and reproduce the factor $\sim 2$ difference between the cases with and without mirrors (shown in Figures 10 and 9, respectively). The effect of the mirror modes is to shift the $\lambda_{\mathrm{e}}$ distribution to lower values of $\lambda_{\mathrm{e}}$ (by a factor $\sim 2-3$, as seen in Figure 11). This can be understood as the electrons experiencing both pitch-angle scattering by whistler waves and trapping by large amplitude mirror modes. Pitch-angle scattering tends to untrap the trapped electrons by taking them into the loss cone of the mirror modes on timescales comparable to the mean pitch-angle scattering time, $\sim \nu_{\text {eff }}^{-1}$. Pitch-angle scattering can also trap the untrapped electrons on similar timescales (see, e.g., Komarov et al. 2016). Thus, the distance $d$ traveled by an electron during a time $\Delta t$ (and, therefore, the estimated value of $\lambda_{e}$ ) should be scaled down by a factor that reflects the fraction of the time that the electrons are untrapped and free to move diffusively.

\footnotetext{
8 We have chosen the interval $t \cdot s=1.5$ to 2.5 so that: (i) the mirror modes in run MW1 are in the fully saturated regime, and (ii) $\Delta t=s^{-1}$ is much larger than the average pitch-angle scattering time, $\sim \nu_{\text {eff }}^{-1}$, of electrons (necessary to assume diffusion). According to Equation (3), $\nu_{\text {eff }}^{-1} \sim 0.1 s^{-1}$, so we can safely assume $d^{2}=\lambda_{\mathrm{e}} v_{\text {th,e }} \Delta t$.
} 


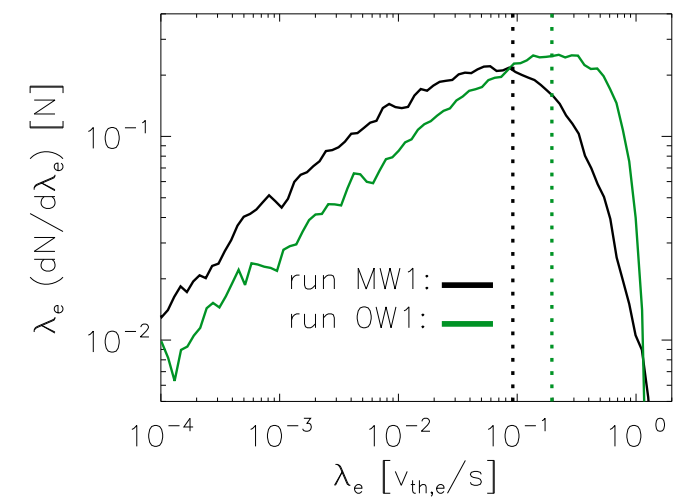

Figure 11. The distribution of electron mean free paths, $\lambda_{\mathrm{e}}\left(d N / d \lambda_{\mathrm{e}}\right)$, for electrons in runs OW1 (only whistlers; green line) and MW1 (whistlers and mirrors; black line), normalized by the total number of particles $(N$; $\left(d N / d \lambda_{\mathrm{e}}\right) d \lambda_{\mathrm{e}}$ is the number of particles with mean free path between $\lambda_{\mathrm{e}}$ and $\left.\lambda_{\mathrm{e}}+d \lambda_{\mathrm{e}}\right) . \lambda_{\mathrm{e}}$ for each particle is calculated measuring the distance $d$ traveled by each electron along $\boldsymbol{B}$ during an interval $\Delta t=s^{-1}$, from $t \cdot s=1.5$ to 2.5, and assuming $d^{2}=\lambda_{\mathrm{e}} v_{\text {th,e }} \Delta t$. The vertical-dotted green and black lines mark the average $\left\langle\lambda_{\mathrm{e}}\right\rangle$ for the OW1 and MW1 runs, respectively.

\section{DISCUSSION AND IMPLICATIONS}

We have used PIC plasma simulations to study the nonlinear evolution of ion and electron velocity-space instabilities in collisionless plasmas. We have focused on instabilities driven by pressure anisotropy with $p_{\perp, j}>p_{\|, j}$. The motivation for doing so is in part that this sign of pressure anisotropy describes the typical conditions found in simulations of lowcollisionality accretion flows onto black holes (Sharma et al. 2006; Riquelme et al. 2012; Foucart et al. 2016). In our calculations, an imposed shear velocity in the plasma amplifies a background magnetic field on a timescale long compared to the cyclotron motion of particles. This drives $p_{\perp, j}>p_{\|, j}$ by the adiabatic invariance of the magnetic moment. The pressure anisotropy in turn drives velocity-space instabilities. The nonlinear, saturated state then depends on how the velocityspace instabilities inhibit the growth of pressure anisotropy.

In order to achieve reasonable scale separation between ions and electrons, we have focused on moderately large values for the mass ratio $m_{\mathrm{i}} / m_{\mathrm{e}}$, and found that for $m_{\mathrm{i}} / m_{\mathrm{e}}=64$ and $m_{\mathrm{i}} /$ $m_{\mathrm{e}}=128$ our results are fairly independent of the mass ratio. Our calculations have focused on the parameter regime $\beta_{\mathrm{i}} \approx \beta_{\mathrm{e}}=1-20$, which is relevant for a wide variety of heliospheric and astrophysical plasmas. In particular, our simulations begin with $\beta_{\mathrm{i}}=\beta_{\mathrm{e}}=20$ but $\beta_{j}$ decreases as the background magnetic field is amplified in time. In this regime the mirror instability is the dominant ion-scale instability (although with a subdominant contribution from the IC instability). As in previous work (Kunz et al. 2014; Riquelme et al. 2015), the mirror instability grows to large amplitudes $\delta B \sim 0.3 B$, even when the background magnetic field is amplified on a timescale long compared to the ion/electron cyclotron periods. In addition to the mirror instability, the anisotropic electrons excite the whistler instability on scales of order the electron Larmor radius, much smaller than the scale of the mirror modes (see Figures 4 and 5).

In the nonlinear saturated state, the ion and electron pressure anisotropies saturate near the thresholds for the corresponding instability, namely mirror and whistler, respectively. Moreover, the magnetic moment decreases in time due to pitch angle scattering by the relevant instabilities (Figures 6(e) and (f)).
More quantitatively, the electron pressure anisotropy in simulations with infinite mass ions (where the ions simply provide a neutralizing charge, but do not excite mirror modes) is well described by the linear theory expectation for the whistler instability (see Figures 3(c) and (d)). For finite mass ratios, however, the electron pressure anisotropy becomes inhomogeneous (Figure 7(a)) and $\left\langle\Delta p_{\|, \mathrm{e}}\right\rangle /\left\langle p_{\|, \mathrm{e}}\right\rangle$ is further reduced by a factor of $\sim 1.5-2$ (Figures $6(\mathrm{c}$ ) and (d)). We attribute this to the effect of the large-amplitude mirror modes on the electrons, which reduce the growth of the perpendicular electron pressure by bunching the electrons into magnetic mirrors on lengthscales comparable to the ion Larmor radius. The obtained ion pressure anisotropy, $\left\langle\Delta p_{\|, i}\right\rangle /\left\langle p_{\|, i}\right\rangle$, is in good agreement with the linear mirror threshold.

We have also used our simulations to compute the mean free path of particles, $\lambda_{j}(j=i, e)$, during the nonlinear stage of the mirror and whistler instabilities. The average mean free path of both ions and electrons is reasonably well described by

$$
\left\langle\lambda_{j}\right\rangle \approx 0.3 \frac{\left\langle\Delta p_{j}\right\rangle}{\left\langle p_{\|, j}\right\rangle} \frac{v_{\mathrm{th}, j}}{q}
$$

where $q$ ( $\left.\equiv s\left|B_{x} B_{y}\right| / B^{2}\right)$ is the growth rate of the magnetic field strength, and $s$ is the shear rate (our shear set up is defined by a fluid velocity $\boldsymbol{v}=-s x \hat{y}) .{ }^{9}$ Physically, this equation describes the balance between pitch-angle scattering by velocity-space instabilities (which limits the pressure anisotropy to $\Delta p_{j}$ ) and driving of the pressure anisotropy by the amplification of the background magnetic field at a rate $q$ (see Equations (1) and (2) and associated discussion).

Equation (6), together with the relevant instability thresholds, provides a deceptively simple prescription for the ion and electron mean free paths in a low collisionality plasma. This in turn provides an upper limit on the thermal conductivity of low-collisionality $\beta_{j} \gtrsim 1$ plasmas. Of course, these results only apply if the mean free path set by velocity-space instabilities is smaller than the Coulomb mean free path for the plasma under consideration.

A second implication of Equation (6) is that a collisionless plasma has a finite viscosity because the particles do not simply free-stream. In our simulations with a background velocity shear, the particles are thus heated by tapping into the background shear, just as in a collisional fluid. We find that the ion and electron heating rates in our simulations are in good agreement with the analytically predicted heating rate by anisotropic viscosity in the limit of a gyrotropic distribution function (Sharma et al. 2007):

$$
\frac{d\left\langle U_{j}\right\rangle}{d t}=-s\left\langle\Delta p_{j}\right\rangle \frac{B_{x} B_{y}}{B^{2}} .
$$

The good agreement between Equation (7) and our numerical heating rates in Figure 8 provides additional support for including this "viscous" heating in models of the thermodynamics of low-collisionality plasmas.

The threshold pressure anisotropy found in our simulations is not exactly appropriate for heliospheric and astrophysical plasmas because in the latter the shear rate is much smaller

\footnotetext{
9 The shear rate in a turbulent plasma, defined as $k \delta v$, can be dominated by small scales, i.e., high wavenumber $k$. However, the shear rate that matters here is related to the timescale for the magnitude of $B$ to change, and will thus typically be dominated by large scale dynamics.
} 
relative to the cyclotron frequency than in our simulations. The astrophysically relevant threshold for the mirror instability is $\Delta p_{\mathrm{i}} / p_{\|, i} \lesssim 1 / \beta_{\mathrm{i}}$, while for the electron whistler instability it is

$$
\frac{\Delta p_{\mathrm{e}}}{p_{\|, \mathrm{e}}} \lesssim \frac{A}{\beta_{\|, \mathrm{e}}^{0.8}} .
$$

Equation (8) is an approximate fit to the whistler instability threshold relevant for both non-relativistic and relativistic electrons, for growth rates $\gamma_{w} \sim 10^{-7} \omega_{c, \mathrm{e}}$; the relativistic calculations are based on numerical solutions of the dispersion relation derived in Gladd (1983) (see Ressler et al. 2015, Appendix B2). The fit is accurate to about $50 \%$ for $\beta_{\|, \mathrm{e}} \simeq 0.1-100$ (note that Gary \& Wang (1996) and Sharma et al. (2007) found a somewhat shallower slope $\propto \beta_{\mathrm{e}}^{-0.55}$ in nonrelativistic calculations over a smaller range of $\beta_{\|, \mathrm{e}}$ ). The coefficient $A$ in Equation (8) depends weakly on electron temperature, varying from $A \simeq 0.125$ for non-relativistic electrons to $A \simeq 0.25$ for $k_{\mathrm{B}} T_{\mathrm{e}} \simeq 10 \mathrm{~m}_{\mathrm{e}} c^{2}$ (relevant to hot accretion flows onto black holes). Finally, in applying Equation (8) to estimate $\left\langle\lambda_{\mathrm{e}}\right\rangle$ (Equation (6)) and $d\left\langle U_{\mathrm{e}}\right\rangle / d t$ (Equation (7)), the reduction in $\left\langle\Delta p_{\mathrm{e}}\right\rangle /\left\langle p_{\|, \mathrm{e}}\right\rangle$ by a factor $\sim 1.5-2$ due to nonlinear mirrors should be included.

The velocity-space instabilities studied in this paper can impact the electron pressure anisotropy, mean free path, thermal conduction, and viscous heating in a wide variety of astrophysical environments, including galaxy clusters, lowluminosity accretion flows onto compact objects, and the solar wind. As a concrete example, we scale our estimate of the electron mean free path to conditions relevant to galaxy clusters using Equations (6) and (8):

$$
\left\langle\lambda_{\mathrm{e}}\right\rangle \sim 10 \mathrm{kpc}\left(\frac{f_{M}}{2}\right)^{-1}\left(\frac{\beta_{\mathrm{e}}}{100}\right)^{-0.8}\left(\frac{T_{\mathrm{e}}}{10^{8} \mathrm{~K}}\right)^{1 / 2}\left(\frac{q^{-1}}{10^{8} \text { year }}\right),
$$

where $f_{M}$ quantifies the suppression of the thermal conductivity due to mirrors. The precise magnetic growth timescale $q^{-1}$ for clusters is uncertain so we have scaled our estimate to about 0.1 of the typical cluster dynamical time. This is likely a modest underestimate at large radii (near the virial radius the timescales are somewhat longer) and a modest overestimate at small radii (in the core the timescales are somewhat shorter). For comparison, the Coulomb mean free path for a Coulomb logarithm of 10 is

$$
\lambda_{\mathrm{C}} \approx 0.4 \mathrm{kpc}\left(\frac{T_{\mathrm{e}}}{10^{8} \mathrm{~K}}\right)^{2}\left(\frac{n}{0.1 \mathrm{~cm}^{-3}}\right)^{-1} .
$$

Note that the whistler mediated mean-free path in Equation (9) is independent of density. It may well be shorter than the Coulomb mean free path at large radii in massive (hot) clusters where densities are typically $\sim 10^{-3}-10^{-2} \mathrm{~cm}^{-3}$. This highlights the importance of velocity-space instabilities for understanding the thermodynamics of the outer parts of massive galaxy clusters.

Our results are also relevant for models of low-luminosity accretion flows onto compact objects. In particular, our results can be incorporated as sub-grid models for electron conduction and heating in numerical simulations of black hole accretion that attempt to directly predict the emission from the accreting plasma (e.g., Mościbrodzka et al. 2014; Ressler et al. 2015).
For concreteness, we note that in the accretion disk context, Equation (7) can be rewritten in terms of the fraction $f_{\mathrm{e}}$ of the total dissipation that goes into the electrons via viscous heating. The total heating rate per unit volume in magnetized accretion disks is given approximately by $-s B_{x} B_{y} / 4 \pi$ (Balbus \& Hawley 1998). As a result, Equation (7) corresponds to

$$
f_{\mathrm{e}} \simeq 0.15\left(\frac{f_{M}}{2}\right)^{-1}\left(\frac{\beta_{\mathrm{e}}}{100}\right)^{0.2},
$$

where we have used Equation (8). Equation (11) predicts an electron heating rate that is a significant fraction of the total dissipation in accretion disks, and is itself only a weak function of the electron thermodynamics. ${ }^{10}$

One limitation of our present study applied to black hole accretion flows is that these environments may be characterized by $T_{\mathrm{e}} \lesssim T_{\mathrm{i}}$, which we have not considered here. Sironi \& Narayan (2015) and Sironi (2015) argued that for $T_{\mathrm{e}} \ll T_{\mathrm{i}}$ the IC instability becomes more prominent than the mirror instability at $\beta_{\mathrm{i}} \lesssim 100$ and that the electric fields associated with the IC instability can transfer energy directly to the electrons, providing a significant source of electron heating. The dominance of the IC instability over the mirror instabilty is not captured by the linear stability calculations we have carried out, but for the electrons these are restricted to non-relativistic plasmas. We also suspect that electron heating by the IC instability is sub-dominant relative to other heating mechanisms (e.g., turbulence and viscous heating) given that for realistic parameters, little of the free energy of the system will reside in the electromagnetic fields associated with the IC waves. Regardless, however, of this subtlety about the physics of the ion-scale instabilities, the electron pressure anisotropy will still be predominantly regulated by the whistler instability, and so the results presented in this paper are applicable for $T_{\mathrm{e}} \ll T_{\mathrm{i}}$ (aside perhaps from the suppression of the electron mean free path by large amplitude mirrors).

We thank Matt Kunz, Lorenzo Sironi, and Alex Schekochihin for useful conversations. MR thanks the Chilean Comisión Nacional de Investigación Científica y Tecnológica (CONICYT; Proyecto Fondecyt Iniciación $\mathrm{N}^{\mathrm{o}}$ 11121145). This work was supported by NSF grant AST 13-33612, a Simons Investigator Award to EQ from the Simons Foundation and the David and Lucile Packard Foundation. DV also acknowledges support from NSF/SHINE grant AGS-1460190 and NASA grant NNX16AG81G. We are also grateful to the UC Berkeley-Chile Fund for support for collaborative trips that enabled this work. This work used the Extreme Science and Engineering Discovery Environment (XSEDE), which is supported by National Science Foundation grant number ACI-1053575.

\section{REFERENCES}

Balbus, S. A., \& Hawley, J. F. 1998, RvMP, 70, 1

Buneman, O. 1993, Computer Space Plasma Physics (Tokyo: Terra Scientific) Chew, G. F., Goldberger, M. L., \& Low, F. E. 1956, RSPSA, 236, 112 Foucart, F., Chandra, M., Gammie, C. F., \& Quataert, E. 2016, MNRAS, 456, 1332

Gary, S. P. 1992, JGR, 97, 8519

\footnotetext{
${ }^{10}$ Sharma et al. (2007) found a somewhat stronger dependence of the electron heating rate on electron temperature, $\propto T_{\mathrm{e}}^{1 / 2}$. The difference lies in our treatment of the whistler instability threshold (see Equation (8) and associated discussion).
} 
Gary, S. P., \& Wang, J. 1996, JGR, 101, 10749

Gladd, N. T. 1983, PhFl, 26, 974

Hasegawa, A. 1969, PhFl, 12, 2642

Hellinger, P., \& Travnicek, P. M. 2008, JGR, 113, A10109

Komarov, S. V., Churazov, E. M., Kunz, M. W., \& Schekochihin, A. A. 2016, arXiv: $1603.00524 \mathrm{v} 2$

Kulsrud, R. M. 1983, in Handbook of Plasma Physics, ed. M. N. Rosenbluth, \& R. Z. Sagdeev (Amsterdam: Elsevier), 115

Kunz, M. W., Schekochihin, A. A., \& Stone, J. M. 2014, PhRvL, 112, 205003 Lyutikov, M. 2007, ApJL, 668, L1

Marsch, E. 2006, LRSP, 3, 1

Maruca, B. A., Kasper, J. C., \& Bale, S. D. 2011, PhRvL, 107, 201101

Mościbrodzka, M., Falcke, H., Shiokawa, H., \& Gammie, C. F. 2014, A\&A, 570, A7

Remya, B., Reddy, R. V., Tsurutani, B. T., Lakhina, G. S., \& Echer, E. 2013, JGRA, 118, 785
Ressler, S. M., Tchekhovskoy, A., Quataert, E., Chandra, M., \& Gammie, C. F. 2015, MNRAS, 454, 1848

Riquelme, M. A., Quataert, E., \& Verscharen, D. 2015, ApJ, 800, 27

Riquelme, M. A., Quataert, E., Sharma, P., \& Spitkovsky, A. 2012, ApJ, 755,50

Schekochihin, A. A., Cowley, S. C., Kulsrud, R. M., Hammett, G. W., \& Sharma, P. 2005, ApJ, 629, 139

Sharma, P., Hammett, G. W., Quataert, E., \& Stone, J. 2006, ApJ, 637, 952

Sharma, P., Quataert, E., Hammett, G. W., \& Stone, J. 2007, ApJ, 667, 714

Sironi, L. 2015, ApJ, 800, 89

Sironi, L., \& Narayan, R. 2015, ApJ, 800, 88

Snyder, P. B., Hammett, G. W., \& Dorland, W. 1997, PhPl, 4, 3974

Southwood, D. J., \& Kivelson, M. G. 1993, JGR, 98, 9181

Spitkovsky, A. 2005, in AIP Conf. Proc. 801 (Melville, NY: AIP), 345

Verscharen, D., Bourouaine, S., Chandran, B. D. G., \& Maruca, B. A. 2013, ApJ, 773, 8 